\title{
On the Complexity of Routing in Wireless Multihop Network
}

\author{
Sonia Waharte \\ University of Bedfordshire \\ Luton, UK \\ Email:sonia.waharte@beds.ac.uk
}

\author{
Alexander Golynski \\ Google Inc. \\ New York, US \\ Email:agolynski@google.com
}

\author{
Raouf Boutaba \\ University of Waterloo \\ Waterloo, Ontario, CA \\ Email:rboutaba@cs.uwaterloo.ca
}

\begin{abstract}
Wireless backbone networks represent an attractive alternative to wired networks in situations where cost, speed of deployment, and flexibility in network design are important. In typical configurations, users connect to wireless routers of the backbone network, which then redirect the traffic to one of the existing network gateways. To improve the network performance, wireless backbone routers redirect their traffic to the network gateways so as to maximize amount of traffic that can be supported by the network. In this paper, we prove that this problem is NP-hard as a result of the wireless interference that is created between geographically close transmission links. We consequently design and investigate the performance of interference-aware algorithms suitable for multi-channel environments against more traditional routing approaches. We evaluate their performance in simulated environments based on data taken from existing networks, and show that interference-based heuristics exhibit advantageous performance in non-uniform deployment.
\end{abstract}

\section{INTRODUCTION}

The number of deployed wireless backbone networks (also referred to as wireless mesh networks) has progressively increased as the possibility of providing network connectivity almost everywhere with minimum deployment time and reduced initial investment represents for many communities and industrial groups an attractive alternative to more expensive wired networks. This gain in flexibility comes at the cost of having to deal with the unreliability inherent to wireless communications. This partly results from environmental interference and partly from the presence of multiple devices competing to simultaneously transmit on the same frequency band. In the design of wireless backbone networks, traffic flows have been considered to be mainly between end users connected to the wireless routers and a set of dedicated network gateways. However, traffic flows can also exist between end users collocated within the same local wireless networks. Applications such as file sharing or voice traffic are very likely to become a non-negligible part of the traffic flows on university or industrial campuses for instance. It is therefore crucial to take advantage of the presence of multiple gateways to achieve a better load distribution and hence improve the overall network performance.

Supported by EPSRC project EP/J012564/1, IRAC at the University of Bedfordshire.

978-1-4577-1379-8/12/\$26.00 (C) 2012 IEEE
The problem we are investigating is to maximize the network utilization, problem which can be reformulated as: how to establish the routing paths from wireless routers to network gateways in order to accept the maximum amount of traffic without congesting the network (which happens when the traffic routed through a path reaches the maximum capacity on one of the links along the path). Wireless routers have the primary role to aggregate traffic from users directly associated to them and to forward the aggregated traffic towards the destination. Network gateways are routers that establish a bridge between different networks, typically between a wired network and a wireless network. It is important to realize that the routing algorithm that determines towards which gateway a wireless router directs its traffic can, in certain cases, lead to a radically different performance. For instance, many routers can be geographically close to one particular gateway. However, sending the traffic of some of them to a more distant but lightly-loaded gateway might lead to a better use of network resources. The routing problem investigated in this paper is effectively a router-to-gateway association problem, which is a type of congestion control problem that can be reduced to minimization of the maximum link utilization.

In this paper, we consider a wireless backbone network with $M$ gateways, $M \geq 2$. We prove that the problem of maximizing the network utilization is NP-hard under the Edge-to-Edge interference model. We then present several interference-aware heuristics and compare them to traditional routing approaches.

The remainder of the paper is organized as follows. In Section II, we present background information and related works. A formal description of the problem we are addressing is presented in Section III. The problem complexity is analyzed in Section IV. Our algorithms are then described precisely in Section V and evaluation results are shown in Section VI. Section VII concludes this paper.

\section{BACKGROUND AND RELATED WORK}

Wireless backbone networks are commonly composed of three types of components: end systems, wireless routers and network gateways [1]. One component may have multiple functions as in community networks for instance, where an end system also acts as a router and relays other users traffic [2] [9] [11]. Traffic between end systems and routers, and 
traffic between routers and gateways are typically transmitted over different network interfaces which do not interfere. In this work, we are interested in studying how to route traffic in the backbone network (between the routers and the gateways). The traffic demands we consider represent, at each router/access point, the aggregate traffic load generated by the end systems associated with this particular router/access point.

One of the main challenges in wireless backbone networks is to efficiently manage the limited channel capacity in order to satisfy users' quality-of-service expectations. Besides the physical data transfer limitations, interference resulting from transmissions over multiple hops (intra-flow and inter-flow interference) [6] can significantly reduce the available throughput. Several solutions have been proposed to address this issue. Routers and end systems can be equipped with multiple interfaces and transmit over multiple channels simultaneously [4]. This approach can result in a significant improvement in network performance. But it also complicates the network management process as it requires efficient router-to-channel assignment algorithms. Another approach is to associate one user to multiple routers (access points) [8] in order to balance the traffic load according to the utilization level of the routers and the data traffic requirements. For instance, unicast traffic can be sent to a router that supports high data rates, whereas broadcast traffic can be sent at a lower data rate to a different router. But in order to be effective, this approach requires some tight synchronization between end systems and routers. Another approach is to take advantage of the presence of multiple gateways. A router can send its traffic to several gateways instead of directing it to a single one. In [12], the authors studied the problem of maximizing the aggregate throughput under fairness constraints and showed that splitting a router's traffic and sending it to several gateways can improve the network capacity as a result of a better load balancing. This, however, comes at the cost of adding complexity in reassembling and reordering data traffics at the gateways. In [12] and [13], it is also assumed that the gateways are always the bottlenecks, which can easily be disproved using the examples shown in Fig. 1 and Fig. 2. In these cases, we can prove that splitting the traffic and sending it to different gateways may not improve the network performance as the central link is the bottleneck. In our work, we consider the more general case of associating routers to gateways when multiple channels are available and we allow gateways to be assigned non-overlapping channels.

\section{Problem Definition}

In this work, our goal is to minimize the maximum link utilization which in turn will result in maximizing the network utilization. We use the following terminology to further describe the problem we are studying.

Definition 1: Given a link $(i, j)$, the interfering set of $(i, j)$ called $I n f_{i j}$ is defined as the group of links for which at least one endpoint is at interference range of $i$ and/or $j$ (including link $(i, j)$ ).

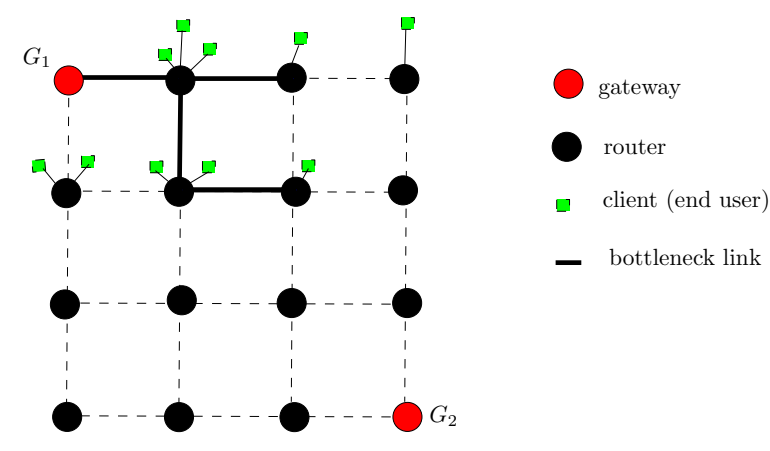

Fig. 1. Case 1: All flows are sent to $G_{1}$. Under the Edge-to-Edge interference model (all nodes at interference distance of a sender and receiver should remain silent for the transmission to be successful), there exist 4 bottleneck links (bold links).

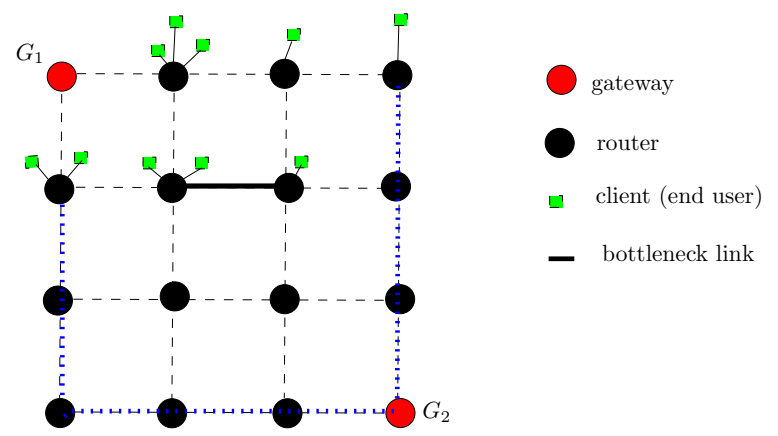

Fig. 2. Case 2: Some of the flows are sent to $G_{2}$ (bold, dashed links). The middle link (bold) remains the bottleneck.

Definition 2: Given a directed graph $G(V, E)$ in which each link $(i, j) \in E$ carries a traffic $f(i, j)$, the link utilization $I_{i j}$ of $(i, j)$ is defined as the sum of the traffics of the links in the interfering set of $(i, j)$ divided by their respective capacities.

$$
I_{i j}=\sum_{(k, l) \in I n f_{i j}} \frac{f(k, l)}{C(k, l)}
$$

A link utilization of 1 means that the link is congested.

Wireless backbone networks present a set of unique characteristics, which opens new possibilities for addressing this goal. First, the presence of multiple gateways enables more efficient load balancing strategies. Indeed, a router can decide to send its traffic to the gateway that offers the best performance in terms of overall link utilization, end-to-end delay, number of nodes supported by each gateway, etc. Second, channel diversity can be exploited by creating connected group of routers operating on different channels, each group being served by one gateway. Another important parameter to account for is the interference between neighboring links operating on the same channel. Consequently, in order to determine the actual availability of a link, it is necessary to account for all the transmissions that can occur at interference range of the link considered. In the following, we refer to the problem of minimizing the maximum link utilization as MIN_MAX_LINK_UTILIZATION. We formulate this 
problem as follows:

Given a connected network topology and a traffic demand vector, determine the load distribution that minimizes the maximum link utilization.

Routers operate on one of the $K$ available channels. Gateways operate on distinct channels, and serve non-overlapping networks. We first start by analyzing the complexity of the above-described problem and demonstrate that in the general case, minimizing the maximum link utilization in wireless networks is NP-hard. We then propose solutions to this problem under different sets of constraints in multi-channel networks.

\section{Min-Max Link Utilization in WMNs: Problem COMPLEXITY}

In this section, we study the following network connectivity problem. Given a set of nodes $V$ on a plane, and the transmission distance $R$ (we use Euclidean distance here), a node $x \in V$ can communicate with $y \in V$ directly if $d(x, y) \leq R$. All pairs of nodes that can communicate form an undirected graph $G(V, E)$. The problem of minimizing the maximum congestion can be stated as follows:

OBJECTIVE: Find a subgraph $G$ ' of $G$ such that

1) $G^{\prime}$ connects all the nodes (spanning tree); the edges of $G^{\prime}$ are called links;

2) the maximum link utilization is minimized

This problem can be seen as a restricted version of $M I N \_M A X \_L I N K \_U T I L I Z A T I O N$, where we assume that all our traffic is symmetric, that is there are no designated sources and sinks, or so called nowhere-zero flow [3]. It also has an additional restriction that all link capacities are 1 in both directions. We show that even in this restricted scenario, the problem of minimizing the maximum utilization is still NP-hard and so, the polynomial time solution for this problem is unlikely to exist. Moreover, we will show that the solution to this problem is also hard to approximate to within factor $47 / 46$ (unless $P=N P$ ).

We study the problem under the Edge-to-Edge interference model. In this model [7], all the nodes at transmission distance of the sender and receiver should remain silent as reflected in the operations of IEEE 802.11 MAC protocol. In 802.11like networks, virtual carrier-sensing is performed between a sender and receiver via the exchange of Request-To-Send (RTS) / Clear-To-Send (CTS) messages. Consequently, all the nodes that receive these messages are blocked therefore avoiding simultaneous transmissions that would result in data collisions.

Theorem 4.1: Under the Edge-to-Edge interference model, $M I N \_M A X \_L I N K \_U T I L I Z A T I O N$ is NP-hard to approximate within factor $47 / 46$.

Proof: We reduce a known NP-complete problem, the Hamilton path problem on grid graphs [5], to our problem. Given a grid graph $H$, we construct an instance of the MIN_MAX_LINK_UTILIZATION, G, given an integer $K$, and $R>0$, such that $H$ has a
Hamilton path with specified endpoints if and only if MIN_MAX_LINK_UTILIZATION of $G$ with transmission distance $R$ is at most $K$.

Definition 3 ([5]): A grid graph is composed of a set of points with integer coordinates, such that two nodes are connected if the Euclidean distance between them is equal to 1 .

Definition 4: A Hamilton path is a path between two vertices of a graph that visits each vertex exactly once.

The instance $G$ can be constructed in two steps: (i) we replace edges of $H$ by the gadget represented in Figure 3; (ii) we replace each of resulting vertices by a city consisting of several nodes that are located very close to each other (distance less than 1/100). Recall that an edge of a graph is just a pair of nodes located with distance $R$ from each other (all edge capacities are 1). A link is an edge on which the traffic is being sent (graph $\left.G^{\prime}\right)$.
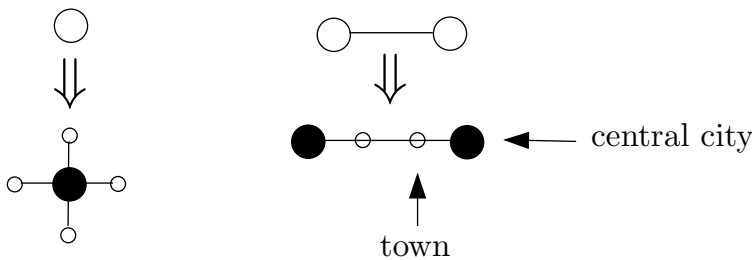

Fig. 3. Gadget used in the reduction: each node in the initial graph $H$ is replaced by a central city surrounded by 4 towns.

We define the size and the number of nodes of these cities later. The transmission radius $R$ can be set to any value between $1 / 3+1 / 50$ and $\sqrt{2} / 3-1 / 50$, so that only neighboring cities can interfere with each other, e.g. see Fig. 4.

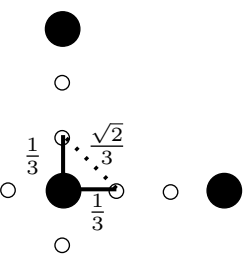

Fig. 4. The transmission radius is set such a node is connected only to its direct neighbors along the $\mathrm{x}$-axis and $\mathrm{y}$-axis (4 at most in total)

Since nodes inside a city are very close to each other, we can show that in the optimal solution to the $M I N \_M A X \_L I N K \_U T I L I Z A T I O N$ in the 2-hop interference model, the links inside a city form a spanning tree.

We say that two cities are connected directly if there is a link between a node $x$ in one city and a node $y$ in the other city. Note that the choice of $x$ and $y$ inside their respective cities does not affect the interference level of any node or link, since the nodes are located very close to each other inside any city (distance less than $1 / 100$ ), that is, if we happen to choose another pair of nodes $x^{\prime}$ and $y^{\prime}, x^{\prime}$ close to $x$ and $y^{\prime}$ close to $y$, for the link, the interference levels on all 
the nodes and edges would not change). We call cities that correspond to the original nodes of $H$ central and the newly created ones towns, edges between towns are called middle edges and edges between central cities and towns are called local edges. We say that two central cities are connected if all the three links in the gadget are present.

We set the size of every central city to $C=1$, except for special cities for which we set the size to be $C+1=2$; and we set the size of all towns to $T=9$. Table I gives the interferences at various edges. The base interference accounts for the edges inside cities, e.g. a town with $T=9$ nodes and 8 edges connecting them creates interference 8 on all of the edges of distance $R$ from it: the neighbor town, the neighbor central city, 5 local edges, and the adjacent middle edge; also each of the 8 edges inside a town interferes with the other 7 edges. The extra interference is calculated based on the maximum possible interference if all the middle and the local edges are all linked. The special interference accounts for the fact that some of the central cities that affect an edge can be special.

Observe that the base interference at local edges is greater than any other type of edges (e.g. $40 \geq 32+8+0$ as compared to the edges inside a special central city), so when solving the $M I N \_M A X \_L I N K \_U T I L I Z A T I O N$ problem, we need to be concerned only with the interference level of the local edges. Also observe that a middle edge affects the interference of the 8 local edges around the two surrounding central cities; while a local edge only affects the other three local edges adjacent to its central city, and one local edge that is adjacent to the next closest central city. Therefore, for each two central cities that are not connected, it is always more beneficial to choose the two local edges to be linked, and the middle edge to be not linked. We set

$$
K=\underbrace{40}_{\text {base }}+\underbrace{4}_{\text {local }}+\underbrace{2}_{\text {middle }}=46
$$

so that we only allow at most two middle edges to interfere with local edge $e$ that is adjacent to a regular central city, and only one middle edge if $e$ is adjacent to a special central city. The solution with $M I N \_M A X \_L I N K \_U T I L I Z A T I O N \leq K$ exists if and only if at least 2 middle edges are not linked around each central city; and at least 3 middle edges are not linked around the two special cities. Therefore, the case of Edge-to-Edge interference is NP-hard.

In fact, we proved a stronger result: we cannot decide between $K=46$ and $K=47$. In other words, a polynomial time approximation algorithm with constant better than $47 / 46$ for Edge-to-Edge interference model does not exist unless $P=N P$.

It is worth noting that in this case, the bound on the approximation ratio has been determined without relying on any assumption on the structure of the network graph considered. However, if we assume that the graphs are connected, the

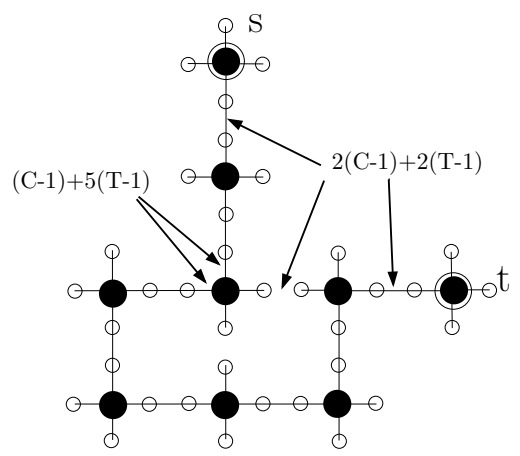

Fig. 5. Edge-to-Edge interference: the base interference for local edges and middle edges is shown as a function of the size of the towns and cities

bound is worst due to the additional interference resulting from the activation of some edges.

Table II gives the interferences at various edges.

Let us set the size of every central city to $C=1$, except for special cities for which we set the size to be $C+1=2$; and we set the size of all towns to $T=5$. The same analysis is performed as for non-connected graphs. The base interference on local edges consequently becomes 20 .

Similarly, we set

$$
K=\underbrace{20}_{\text {base }}+\underbrace{4}_{\text {local }}+\underbrace{2}_{\text {middle }}=26
$$

so that we only allow at most two middle edges to interfere with local edge $e$ that is adjacent to a regular central city, and only one middle edge if $e$ is adjacent to a special central city. Consequently, under the constraint that the graph is connected, a polynomial time approximation algorithm within constant better than $27 / 26$ for the Edge-to-Edge interference model does not exist unless $P=N P$.

Note that this proof can be extended to other interference models in a similar manner. In the following section, we propose some centralized solutions to $M I N \_M A X \_L I N K_{-} U T I L I Z A T I O N$ for a different set of constraints on the routing paths.

\section{Algorithms for Routers to Gateways ASSOCIATION}

We designed several algorithms to associate each router to exactly one gateway. Given the static nature of the network and the specific traffic characteristics, a centralized approach appears more effective and less resource-consuming than a distributed approach.

We proposed two interference-based heuristics that we compared against more traditional routing approaches based on geographical considerations and traffic load. We categorize the algorithms as follows:

- Geographic approaches: Routers are assigned to gateways based on their proximity either in terms of hops (Shortest path algorithm), or in terms of Euclidean distance (Voronoi algorithm).

- Load-balanced approaches: Routers are assigned to gateways so that the traffic load oriented towards the gateways 


\begin{tabular}{|l|l|l|}
\hline Type & Base Interf. & $\begin{array}{l}\text { Max Extra Interf. } \\
+ \text { Max Special Interf. }\end{array}$ \\
\hline inside a special central city & $4(T-1)+(C-1)=32$ & $8+0$ \\
inside a town (if $T \geq 2)$ & $(T-1)+(C-1)+(T-2)=15$ & $6+1$ \\
local edge & $(C-1)+5(T-1)=40$ & $8+1$ \\
middle edge & $2(C-1)+2(T-1)=16$ & $8+2$ \\
\hline
\end{tabular}

TABLE I

INTERFERENCE TABLE FOR NON-CONNECTED GRAPHS

\begin{tabular}{|l|l|l|}
\hline Type & Base Interf. & Min Extra Interf. \\
\hline inside a special central city & $4(T-1)+(C-1)=16$ & 5 \\
inside a town (if $T \geq 2)$ & $(T-1)+(C-1)+(T-2)=7$ & 3 \\
local edge & $(C-1)+5(T-1)=20$ & 4 \\
middle edge & $2(C-1)+2(T-1)=8$ & 2 \\
\hline
\end{tabular}

TABLE II

INTERFERENCE TABLE FOR CONNECTED GRAPHS

is distributed as uniformly as possible (Load-based and Node-based Voronoi algorithms).

- Interference-based approaches: Routers are assigned to gateways so that inter-node interference is minimized (Forces-based and Potential-based algorithms).

\section{A. Geographic approaches}

Shortest paths: A router is associated to the closest gateway in terms of hops (on a graph it corresponds to the number of edges of unit weight between two nodes). If several gateways are at the same distance from the router considered, one is picked at random.

Euclidean Voronoi: A node is associated to the closest gateway (geographically).

\section{B. Load-balanced approaches}

Load-adaptive Multiplicatively Weighted Voronoi: for each gateway $p$ and for each node $X$ we compute the distance $d(p, X) w(p)$ where $d(p, X)$ is the Euclidean distance and $w(p)$ is a weight. $w(p)$ is computed as follows: $w(p)=\sum \delta(i, p) L(i) / \sum L(i)$ with $L(i)$ is the load at node $i$ and $\delta(i, p)=1$ if node $i$ is associated with gateway $p$, 0 otherwise. A more heavily loaded gateway consequently has a greater weight. At each iteration, a router (randomly chosen among the ones at shortest distance from a gateway) is associated to a gateway and the weight of the remaining routers (not already assigned) is recomputed.

Node-adaptive Multiplicatively Weighted Voronoi: Assuming a unit traffic load, i.e. that $L(i)=1$ for all $i$, we apply the same algorithm as for the Load-adaptive Multiplicatively Weighted Voronoi clustering.

\section{Interference-based approaches}

Forces-based algorithm: Each node has a charge $-f_{i}$, that corresponds to its traffic demand. The rationale behind this setting is that the greater the load at a node, the more resource it consumes. Therefore, other neighboring nodes would have to compete more to access the medium, which might impact their performance. A better load balancing avoiding the congested zones would consequently result in a better nework performance. We model this competition for network resources by repulsive forces. Since traffic flows are directed towards gateways, the gateways exert an attractive force on the routers. Each gateway has a charge $-g_{i} \sum f_{i} / \sum_{i} g_{i}$, where $g_{i}$ is the available bandwidth. The gateways with higher bandwidth consequently have a greater attraction force. For each router $i$, we calculate the force applied to it, which corresponds to the sum of all the repulsive forces exerted by the remaining routers $\left(\sum f_{i} f_{j} / d(i, j)^{2} \overrightarrow{u_{j i}}\right)$ plus the attractive forces from the gateways, with $d(i, j)$ the number of hops between router $i$ and $j$. The sum of these forces results in a force that points towards a direction along which a router should direct its traffic. The gateway that is the closest to this direction is selected by the router as destination.

Potential-based algorithm: We use the same underlying idea to derive this algorithm as the one used for the Forcesbased algorithm except that we assign to each edge $(i, j)$ a weight called potential $(i, j)$ which represents the difference of potentials between the two endpoints. For edge $(i, j)$, $\operatorname{potential}(i, j)=\left\|-f_{i}-f_{j}\right\| / d(i, j)$, with $-f_{i}$ and $-f_{j}$ the traffic demands of node $i$ and node $j$ respectively. The potential on each edge therefore reflects the intensity of the traffic load it is susceptible to carry. Edges with high potential should therefore be avoided. The gateways are interconnected by wires of infinite capacity which can be represented on a graph by edges of weight 0 . We then run Kruskal's algorithm to define the minimum spanning tree therefore removing the edges with high potential. This defines the gateway a router should send its traffic to.

\section{Simulation Results}

We evaluated the performance of our algorithms under different scenarios. We primarily focused on their performance in terms of link utilization and load balancing. 


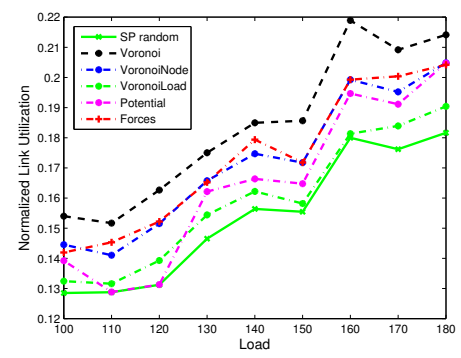

Fig. 6. STD of the Link Utilization at each gateway with Increasing Traffic Load

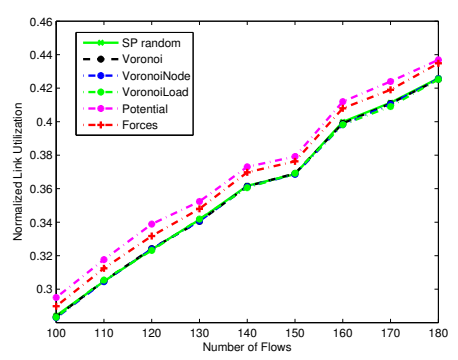

Fig. 7. Average Link Utilization at each gateway with Increasing Traffic Load

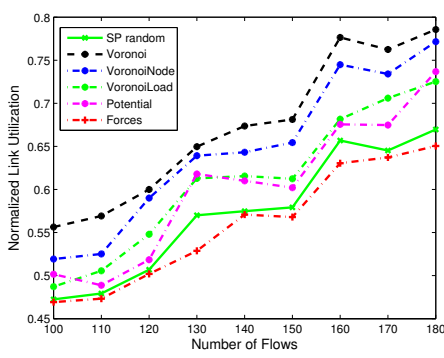

Fig. 8. Max Link Utilization at each gateway with Increasing Traffic Load

\section{A. Simulation Environment}

To solve the linear programs, we used the default algorithm provided by Matlab based on the simplex method. We assume that a central entity is responsible for the route computation and can efficiently transmit this information to the routers with minimum overhead. For simplification, we consider a 2-hop interference model, i.e. all the nodes at transmission range of the sender and receiver should remain silent for the data transmission to be successful. We let the investigation of more complex interference models as future work.

We used the topology of an existing deployed network from the city of Chaska, Minnesota [10] to evaluate the performance of the algorithms.

\section{B. Chaska network}

The network is composed of 195 nodes non uniformly distributed. As no information on the transmission range and on the actual locations of the network gateways were available, we performed some tests and set the transmission to $230 \mathrm{~m}$ so as to guarantee that the whole network is connected. The gateways have been placed as uniformly as possible with a preference for the densest areas (in terms of number of neighboring routers). Following the guidelines in actual network design, we set the number of gateways to 10 such that one gateway serves on average around 20 routers. We also fixed the link capacities at $1 \mathrm{Mbps}$ at the beginning of the simulations.

We can observe in Fig. 6, Fig. 7 and Fig. 8 that the router-togateway allocation using the Forces-based algorithm still performs the best overall. The geographic-based approaches based on Euclidean distances are more sensitive to geographical disparities consequently leading to the poorest performance. The Potential-based and the Shortest-path algorithms alleviate this problem by considering the number of hops between routers instead of Euclidean distances. The Shortest-path algorithm achieves the best load distribution as a consequence of the uniform distribution of the gateways and source routers.

\section{CONCLUSION}

Wireless backbone networks can offer a viable and costeffective solution to the last-mile connectivity issue. However, the unreliability of the wireless transmission medium and limited transmission capacities require careful handling in order to optimize the network utilization.

By exploiting the properties inherent to the wireless backbone networks, we showed that improvements in terms of link utilization and load balancing can be achieved. In particular, the deployment of multiple gateways and the possibility to choose one (or several) of them to direct the traffic to can greatly improve the network utilization.

Our contributions are the following. We showed that the problem of minimizing the maximum link utilization in wireless backbone networks is NP-hard. In fact, we proved that the solution to this problem is hard to approximate within factor $47 / 46$ for the Edge-to-Edge interference model does not exists unless $P=N P$. We then designed several heuristics and evaluated them under different constraints. We showed that depending on the metric evaluated (average link utilization, maximum link utilization, standard deviation), disparities in terms of performance can occur. But overall our interferenceaware Forces-based algorithm exhibited the best performance in the case scenario considered.

\section{REFERENCES}

[1] I.F. Akyildiz and X. Wang. Wireless mesh networks: a survey. Computer Networks, 47:445-487, 2005.

[2] CuWin. http://cuwireless.net/.

[3] J. Gross and Y. Yellen. Handbook of Graph Theory. CRC Press.

[4] S. Huang, W. Wang, Z. Zhang, and J. Zhou. On the capacity of multiradio multi-channel multi-gateway multi-packet reception wireless mesh networks. Journal of Computational Information Systems, 2012.

[5] A. Itai, C.H. Papadimitriou, and J.L. Szwarcfiter. Hamilton paths on grid graphs. Journal on Computing, 1(4):676-686, 1982.

[6] Jangeun Jun and M.L Sichitiu. The nominal capacity of wireless mesh networks. IEEE Wireless Communications, 10(5):8-14, Oct. 2003.

[7] M. Kodialam and T. Nandagopal. The effect of interference on the capacity of multi-hop wireless networks. In IEEE Symposium on Information Theory (ISIT), July 2004.

[8] D. Lee, G. Chandrasekharan, and P. Sinha. Optimizing broadcast load in wireless mesh networks with dual association. In First IEEE Workshop on Wireless Mesh Networks, 2005.

[9] Equal Access Community Internet NetEquality. http://www.netequality.org/.

[10] Chaska Wireless Network. http://chaska.net.

[11] MIT ROOFNET. http://pdos.csail.mit.edu/roofnet/.

[12] Lakshmanan S., Sivakumar R., and Sundaresan K. Multi-gateway association in wireless mesh networks. Ad Hoc Networks, 7(3):622 637, 2009.

[13] H. Tokito, M. Sasabe, G. Hasegawa, and H. Nakano. Routing method for gateway load balancing in wireless mesh networks. In Proceedings of the 2009 Eighth International Conference on Networks, ICN '09, pages 127-132, Washington, DC, USA, 2009. IEEE Computer Society. 